\title{
Sparganosis of the Unilateral Breast: A Case Report
}

\author{
Hyung Suk Kim¹, Man Sik Shin', Chang Jong Kim¹, Sun Hyung You', Yong Hwa Eom¹, Tae Kyung Yoo', \\ Ahwon Lee ${ }^{2}$, Byung Joo Song', Byung Joo Chae ${ }^{1, *}$ \\ ${ }^{1}$ Department of Surgery, ${ }^{2}$ Department of Hospital Pathology, Seoul St. Mary's Hospital, Catholic University of Korea College of Medicine, \\ Seoul 06591, Korea
}

\begin{abstract}
Sparganosis is a parasitic infection caused by the sparganum, the plercercoid of the genus Spirometra. The preoperative diagnosis of breast sparganosis is difficult in most cases because it is a rare parasitic infection less than $2 \%$ of all cases. We report a 62-year-old woman case of breast sparganosis that were confirmed by surgical removal of worms from the right breast. The radiologic images of the patient also revealed characteristic features of breast sparganosis. The patient described the migrating palpable breast mass, which strongly suggested the possibility of breast sparganosis. The treatment of choice and confirmative diagnosis for sparganosis are complete surgical extraction of the sparganum irrespective of infected site. Inspection of the mass site with detailed medical history and radiological examinations are important for preoperative diagnosis of sparganosis patients.
\end{abstract}

Key words: Spirometra, sparganosis, ultrasound, mammography, breast, surgical extraction

\section{INTRODUCTION}

Sparganosis is an uncommon parasitic infection that is caused by tapeworms belonging to the genus Spirometra, specifically, its plerocercoid larva [1]. Sparganosis has been described from all over the world. However, case reports of patients are most prevalent in East Asia. Consuming undercooked snakes or frogs, drinking contaminated water, or coming to contact with infected skin by an open wound are just some of the ways that can begin the infection process [2]. It occurs usually in subcutaneous tissues of the patients, which then migrate to other tissues. Major symptoms of the patients with the sparganum include subcutaneous mass and obscure pain [3]. In humans, most common infection sites are abdominal wall and viscera, urogenital organs, extremities, central nervous system, chest, orbital region, neck, and oral cavity [4]. Cases of breast sparganosis is rare, representing less than $2 \%$ of all cases [5]. In most cases, the preoperative diagnosis of sparganosis is difficult, since it is a rare parasitic disease. Here, we report a case of surgically confirmed sparganosis from the

- Received 24 December 2016, revised 6 June 2017, accepted 15 June 2017.

*Corresponding author (bjchae@gmail.com)

(c) 2017. Korean Society for Parasitology and Tropical Medicine

This is an Open Access article distributed under the terms of the Creative Commons Attribution Non-Commercial License (http://creativecommons.org/licenses/by-nc/4.0) which permits unrestricted non-commercial use, distribution, and reproduction in any medium, provided the original work is properly cited. right breast as well as its clinical, radiological, and pathological findings.

\section{CASE RECORD}

A 62-year-old woman came to our outpatient clinic, displaying a palpable right breast lump for 4-5 months. She experienced obscure discomfort in her right breast, without any nipple discharge or systemic symptoms. Recently, she experienced itching sensation of breast mass lesion as well. Physical examinations revealed a mobile and firm mass, within the normal overlying skin, without any palpable axillary lymph nodes. While she had often travelled to Southeast Asian countries, but she never ate snakes or frogs. However, while she was traveling, she could have taken impure water. Also, she was fond of raw beef, and she ate it regularly.

She visited a local clinic, and a core needle biopsy was performed. She came to our hospital. We suspected a parasitic infection and observed small fragments of worms on the histopathology slide. Mammography showed that the upper-inner quadrant of her right breast had a tubular lesion, about $5.5 \mathrm{~cm}$ in diameter. Breast ultrasonography also showed multiple track-like tubular lesions, about $5.3 \mathrm{~cm}$ diameter in the upper inner quadrant of her right breast and $4.0 \mathrm{~cm}$ diameter in the lower inner quadrant of her right breast (Fig. 1). The chest CT image demonstrated multiple, variable-sized, nodular, or tu- 

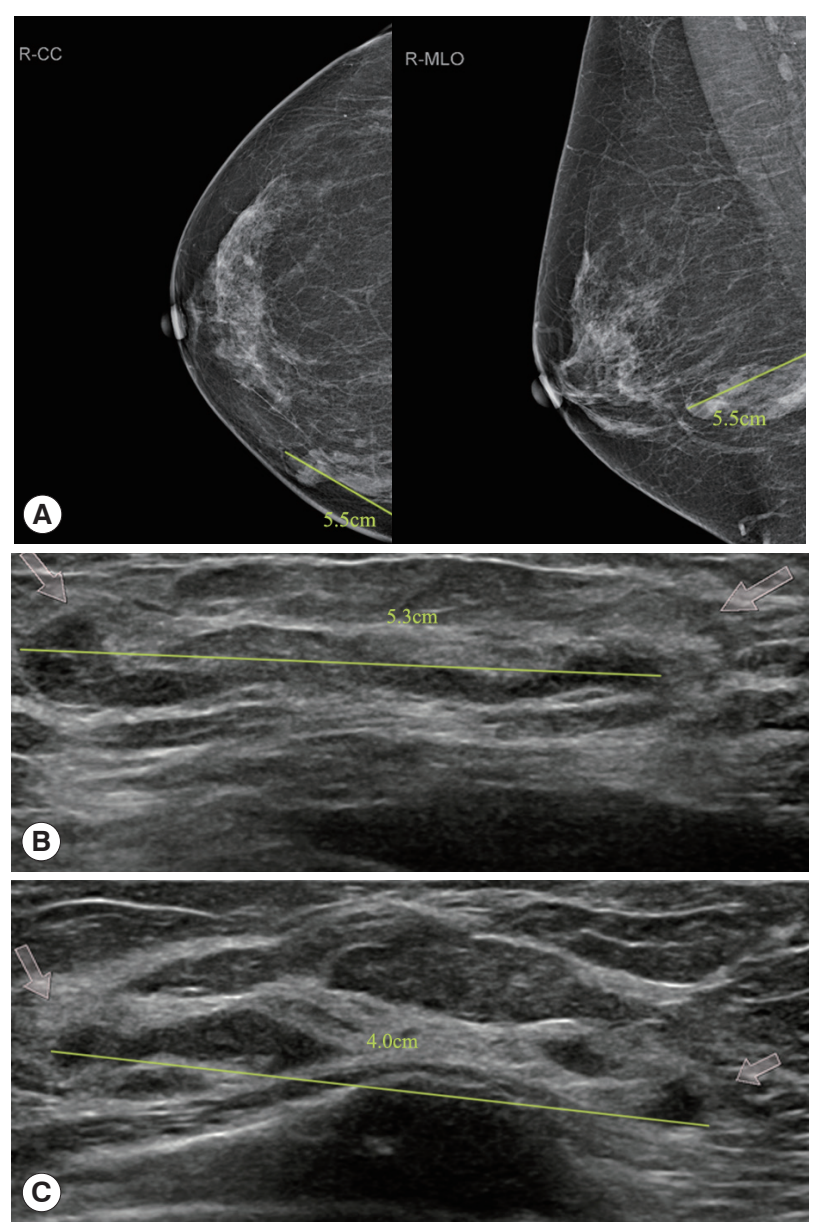

Fig. 1. Breast sparganosis in a 62-year-old woman with a palpable mass in the right breast. (A) Breast mammography reveals a $5.5 \mathrm{~cm}$ extent tubular lesion in the upper inner quadrant of the right breast. $(\mathrm{B}, \mathrm{C})$ Breast ultrasonography shows a $5.3 \mathrm{~cm}$ extent multiple track-like tubular lesions in the upper inner quadrant and a $4.0 \mathrm{~cm}$ lesion in the lower inner quadrant of the right breast.

bular lesions in the medial aspect of the right breast, suggesting sparganosis. Abdominal, pelvic, and brain CT were taken and there were no significant abnormalities. During the operation, 4 pieces of a worm were visually identified (Fig. 2). The histopathologic findings revealed characteristic features of a sparganum and foreign body reaction in the excised breast parenchyme (Fig. 2). The patient's symptoms were relieved after the removal of the breast mass. Anti-sparganum specific IgG level measured 1.22 by ELISA in patient's serum. It was significantly higher than an uninfected control $(0.22)$. However, the anti-sparganum specific IgG level was not examined after the operation.

\section{DISCUSSION}

Sparganosis is a parasitic infection caused by the plercercoid of the genus Spirometra. Sparganosis is more commonly found in the Far East, including China, Japan, and Korea [6]. Human infections occur from drinking water containing cyclops contaminated with procercoids or eating raw frogs and/or snakes. Our patient used water from a restaurant while she was traveling, and that was one instance when she could have taken impure water. The sparganum can migrate various tissues, including the subcutaneous tissues and muscles [2]. It can even migrate to the brain, abdominal wall, extremities, pleura, and bones; however, breast involvement is less than $2 \%$ of all cases [5].

In radiologic images, sparganosis most often appears as a nodular lesion. Therefore, it is difficult to distinguish it from soft tissues. Breast sparganosis presents with clinical symptoms that include palpable breast mass which may bring obscure pain, due to migration of the worm. However, peripheral blood eosinophilia and inflammatory reactions are rarely observed [7]. To rule out malignancy of the breast, a core needle biopsy is required in breast sparganosis. Our patient experienced a subcutaneous mass, vague pain, and itching sensation at the right breast. We found 4 pieces of a worm in the glandular mammary tissue of the right breast. The mammographic and ultrasonographic findings of breast sparganosis can be useful, for preoperative diagnosis and patient management [8]. Mammographic findings usually show a tapeworm-like structure; lobular or tubular lesion in the fat layer [8]. Ultrasonographic findings of sparganosis show several elongated tubular hypoechoic structures [2]. Pathology findings usually reveal foreign body reactions, including granulomatous inflammations along a track-like, elongated cavity, through which the sparganum passed [8]. In our patient, the removed sparganum was histologically characterized by eosinophilic tegument and longitudinal smooth muscle bundles in the loose internal stroma. Multiple cystic spaces in the breast parenchyme were surrounded by foreign body reactions with multinucleated giant cells and chronic inflammatory cells, such as plasma cells, lymphocytes, and a few eosinophils.

Surgical extraction is the confirmative diagnosis for sparganosis. For treatment of sparganosis, medication therapy, including praziquantel, is reported to have only limited effects [9]. Our patient was operated with complete surgical removal of breast masses with 4 pieces of a sparganum worm. Symp- 

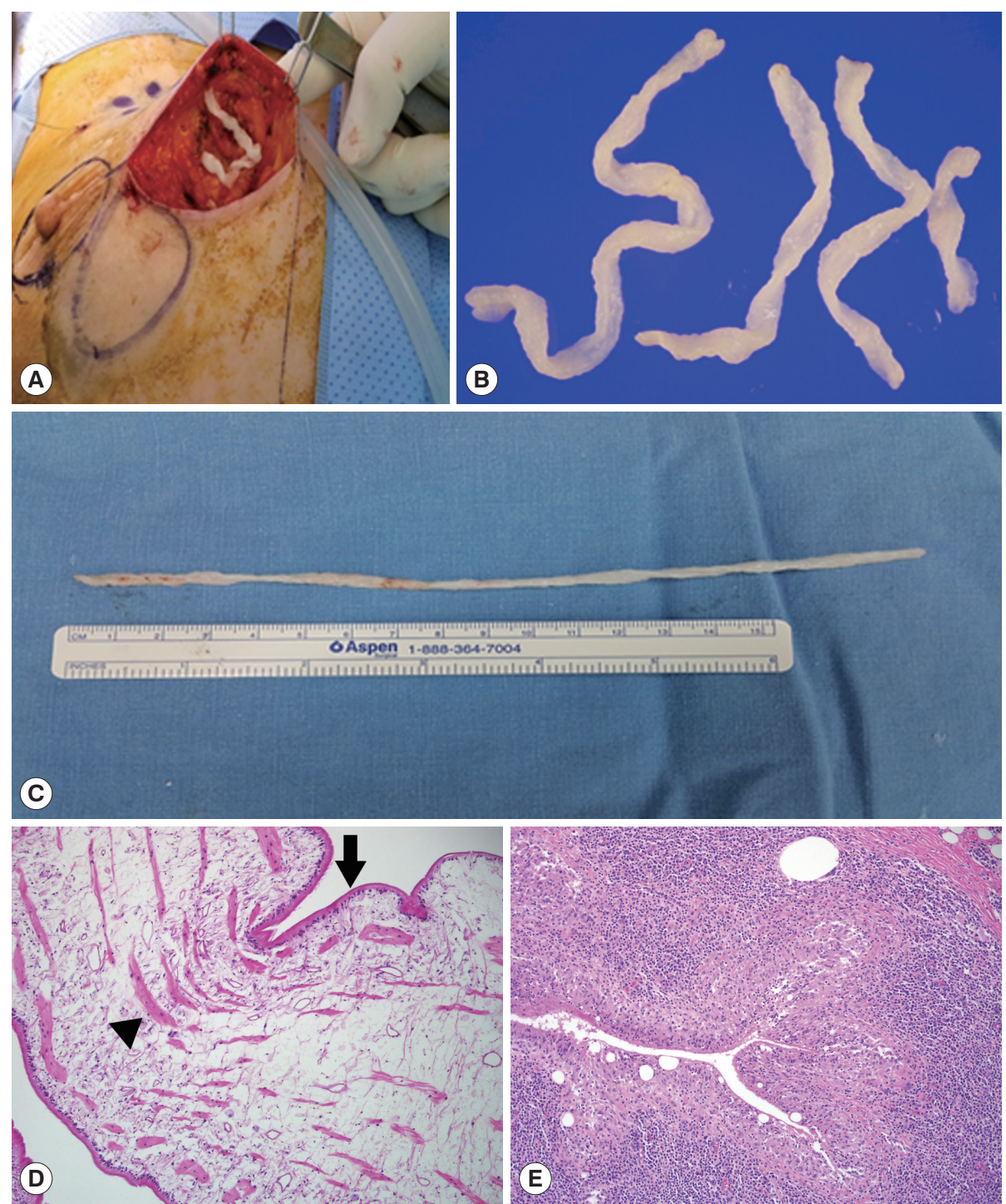

Fig. 2. Gross appearance of the ivory-white sparganum worm and pathologic findings. (A) A sparganum worm is visible in the subcutaneous fat layer under the overlying skin incision. (B, C) An ivory-white worm from the right breast, 3 pieces (B) and another piece (C) confirming the diagnosis of sparganosis. (D) Longitudinal section of the spargaum showing an eosinophilic tegument (arrow) and longitudinal muscle fibers (arrow head) in a loose internal matrix (x100). (E) Cystic spaces surrounded by foreign body reactions and chronic inflammatory cell infiltrates, such as plasma cells, lymphocytes, and eosinophils (x100).

toms of the patient improved after the removal of the breast mass. Conclusively, we report a case of breast sparganosis that were confirmed by complete surgical excision. For the exact diagnosis, an inspection of mass site and detailed medical history and radiologic examination are important in preoperative diagnosis of sparganosis.

\section{CONFLICT OF INTEREST}

The authors declare that they have no competing interest.

\section{REFERENCES}

1. Park JH, Chai JW, Cho N, Paek NS, Guk SM, Shin EH, Chai JY. A surgically confirmed case of breast sparganosis showing characteristic mammography and ultrasonography findings. Korean J Parasitol 2006; 44: 151-156.

2. Choi SJ, Park SH, Kim MJ, Jung M, Ko BH. Sparganosis of the breast and lower extremities: sonographic appearance. J Clin Ultrasound 2014; 42: 436-438.

3. Yoon HS, Jeon BJ, Park BY. Multiple sparganosis in an immunosuppressed patient. Arch Plast Surg 2013; 40: 479-481. 
4. Chang KH, Cho SY, Chi JG, Kim WS, Han MC, Kim CW, Myung H, Choi KS. Cerebral sparganosis: CT characteristics. Radiology 1987; 165: 505-510.

5. Hong SJ, Kim YM, Seo M, Kim KS. Breast and scrotal sparganosis: sonographic findings and pathologic correlation. J Ultrasound Med 2010; 29: 1627-1633.

6. Sim S, You JK, Lee IY, Im KI, Yong TS. A case of breast sparganosis. Korean J Parasitol 2002; 40: 187-189.

7. Chan AB, Wan SK, Leung SL, Law BK, Lai DP, Ip M, Tse GM, Suen MW. Sparganosis of the breast. Histopathology 2004; 44:
510-511.

8. Song EJ, Sohn YM, Ryu KN, Min SY, Shin SH, Park YK. Breast sparganosis and incidentally detected subcutaneous and intramuscular sparganosis at several sites: case report and literature review. Jpn J Radiol 2015; 33: 225-228.

9. Lee JH, Kim GH, Kim SM, Lee SY, Lee WY, Bae JW, Shin KS, Hwang KK, Kim DW, Cho MC. A case of sparganosis that presented as a recurrent pericardial effusion. Korean Circ J 2011; 41: $38-42$. 